American Medical Journal 2 (1): 47-50, 2011

ISSN 1949-0070

(C) 2011 Science Publications

\title{
Efficacy of Intra Uterine Insemination in the Treatment of Infertility
}

\author{
Nabila Kamil Yaaqoub and Bushra Mohammed Majed \\ Department of Gynecology and Obstetrics, Faculty of Medicine, \\ University of Tikrit, P.O. Box 42, Tikrit Salahuddin, Iraq
}

\begin{abstract}
Problem statement: To assess efficacy of IUI in treatment of infertility. Tikrit teaching hospital. Approach: 42 infertile couple underwent standard evaluation for infertility, woman given medication to stimulate development of mature follicles, washed semen specimen placed in uterine cavity using sterile flexible catheter, coincide with ovulation time. Results: Of 42 infertile couples pregnancy occur in 26(61.9\%), (19.1\%) in primary and (42.9\%) in secondary infertility, $(21.3 \%)$ of them was male infertility and $(40.6 \%)$ of unexplained infertility. Pregnancy was less with prolong duration of infertility (35.7\%) with 3-5 years, $(21.3 \%)$ with 5-7 years and (4.8\%) with 8 and more years of infertility. Conclusion: IUI is less expensive, less invasive and effective in treatment of couples with unexplained or male infertility.
\end{abstract}

Key words: Male infertility, mature follicles, Insemination

\section{INTRODUCTION}

Infertility is defined as failure to achieve pregnancy for one year or more without.

Use of contraception during the child bearing period (Stephen and Chandra, 2000).

Infertility affects one in seven couples (Temploton, 1995).

Affects approximately $10-15 \%$ of couples (Stephen and Chandra, 2000).

In addition to its economic costs ,infertility has a major psychological impact. Oddene et al. (1999) report that infertile had depressive and anxiety symptoms four times more frequently than fertile woman.

Two common treatments for infertility are induction ovulation and intrauterine insemination (Voorhis et al., 1997).

Super ovulation and IUI are used alone or in combination for treatment of unexplained infertility , male factor infertility and other cases of infertility in which women has obstructed genital tract and some ovarian function (Voorhis et al., 1997). Table 1 lists types and causes of infertility.

Oral clomiphene citrate a popular choice for treatment of unexplained infertility, is in expensive, require little clinical monitoring and thought to correct sub fertile ovulatory dysfunction (Royal College of Obstetricians and Gynaecologists, 1998).

Antioestrogenic effect of clomiphene citrate on the endometrium, uterine blood flow, the quality of cervical mucus could affect its ability to enhance pregnancy rates (Bilijan et al., 1999).

IUI is the theraputic process of placing washed spermatozoa transcervically into the uterine cavity for treatment of infertility, IUI theoratically allow arelatively higher number of motile spermatozoa to reach acolyte (Abdelkader and Yen, 2009).

This technique has recently joined the list of procedures licensed by Human fertilization and Embryology Authority in the U.K. and its one of the most widely used forms of assisted reproductive technology (Andersen et al., 2008). Table 2 tabulates the pregnancy rates under different types of infertility.

A prolonged duration of infertility has been proposed as indication for use of assisted reproductive technology (Brosens et al., 2004).

Despite increase in use of assisted reproductive technologies They still costy, invasive (7), IVF is associated with undesirable out comes these include preterm delivery leading to increase risk of new born prematurity and its can comitant cost and morbidity and increase rate of multiple gestation (Keddy et al., 2007).

The pregnancy rate of IUI is repotted to 10-20 (7), based on etiology of infertility, the highest rates were reported when IUI was used in patient with an ovulation who were undergoing ovulation induction therapy at time of IUI, male factor infertility and unexplained infertility, in patient with endometrosis , pregnancy rate were the lowest (Ahinko-Hakamaa et al., 2007).

Corresponding Author: Nabila K. Yaaqoub, Department of Gynecology and Obstetrics, Faculty of Medicine, University of Tikrit, P.O. Box 42, Tikrit Salahuddin, Iraq 
The general process of artificial insemination has been used to treat a variety of physiological and psychological male and female infertility disorders (Omloelet et al., 2003).

Factors that may influence IUI outcome include the use of ovulation induction agents, semen analysis parameters, techniques used for sperm preparation and the timing and number of inseminations (Klein et al., 2007).

IUI is contra indicated in women with cervical artesian, cervicitis, endometritis and bilateral tubal obstruction and in most cases of amenorrhea or severs oligospermia (Crosignani and Maggiore, 2009).

\section{MATERIALS AND METHODS}

This was a prospective study, a total of 42 infertile couples were seen in one year (April 2009 to April 2010) and each couple underwent a standard evaluation for infertility, including semen analysis in man according to standardized methods endometrial biopsy HSG and laparoscopy in women (Overstreet, 1997).

The women given medication to stimulate ovulation by 75 IU of follicle-stimulating hormones, intramuscularly daily from day 3 to day 7 , on day 8 ultrasonography was repeated and daily administration of FSH was continued if necessary until follicle reach $18 \mathrm{~mm}$ (Average of two dimension). Then $10000 \mathrm{IU}$ of human chorionic gonadotrophins was administered intramuscularly.

A semen specimen washed in the laboratory called (sperm processing or sperm washing). A sperm separated from other component of semen and concentrated in small volume.

Prior to IUI it's necessary to remove seminal plasma to avoid prostaglandin induced uterine contractions, insemination will unprocessed semen is also associated with pelvic inflection (Boomsma et al., 2007).

The majority of published studies, insemination are done 32-36hr following HCG administration (Crosignani and Maggiore, 2009).

Speculum is pleased in the vagina and cervical area is gently cleaned.

The washed specimen of highly motile sperm is pleased in uterine cavity using sterile, flexible catheter.

\section{RESULTS}

The age of couples in our study ranged from (2142) years, $15(35.7 \%)$ with primary infertility and 27 $(67.3 \%)$ with secondary infertility.
Table 1: Type and cause of infertility

\begin{tabular}{ll}
\hline Type & No. $\%$ \\
\hline Primary & $15(35.7)$ \\
Secondary & $27(64.3)$ \\
Total & $42(100)$ \\
Cause & \\
O'Infertility & $16(38.1)$ \\
Unexplained & $22(52.4)$ \\
Unexplained & $4(9.5)$ \\
Total & $42(100 \%)$ \\
\hline
\end{tabular}

Table 2: Pregnancy rate

\begin{tabular}{llrrr}
\hline Type of infertility & & No. & $\%$ & Total \\
\hline Primary & Yes & 8 & 19.1 & \\
& No & 7 & 16.7 & \\
Secondary & Yes & 18 & 42.9 & \\
& No & 9 & 21.3 & \\
Total & & 42 & 100.0 & \\
Duration of infertility & & & & \\
3-5 years & Yes & 15 & 35.7 & 19 \\
& No & 4 & 9.5 & \\
5-7 years & Yes & 9 & 21.3 & 14 \\
& No & 5 & 12.0 & \\
8-11 years & Yes & 2 & 4.8 & 9 \\
& No & 7 & 16.7 & \\
Total & & 42 & 100.0 & 42 \\
\hline
\end{tabular}

Table 3: Pregnancy out come

\begin{tabular}{lcr}
\hline Live birth & No. & $\%$ \\
\hline At term & 18 & 69.3 \\
Preterm & 3 & 11.5 \\
Still birth & 0 & 0.0 \\
Spontaneous abortion & 4 & 15.4 \\
Ectopic pregnancy & 1 & 3.8 \\
Total & 26 & 100.0 \\
\hline
\end{tabular}

$16(38.1 \%)$ of them has $\sigma^{\prime}$ infertility, $22(52.4 \%)$ with unexplained infertility, $4(95 \%)$ of endometriosis.

Pregnancy rate significantly increase in secondary infertility and unexplained infertility and in shorter duration of infertility Table 2 .

\section{DISCUSSION}

Intra uterine insemination of sperm can potentially enhance pregnancy rates by helping to overcome the cervical barrier. Observation data suggest three fold increases in pregnancy rates with IUI alone, with further increase in concomitant ovarian stimulation. (Bhattacharya et al., 2008).

In our study pregnancy rate was $(61.9 \%),(19.1 \%)$ in primary infertility and $(42.9 \%)$ in secondary infertility and $(21.3 \%)$ of them was male infertility and $(40.6 \%)$ of unexplained infertility.

In cases of unexplained infertility, the early start of IUI is the line with recommendation of progression from low tech to high tech treatment (Collins, 2003). 
In our study pregnancy rate was lower in infertility couples with long duration of infertility, (35.7\%) with 3-5 years of infertility, (21.3\%) with 5-7 years of infertility and (4.8\%) in couples of 8 and more years of infertility.

Regarding the prenatal outcomes of IUI conceptions. Gandoin et al. (2003) report that ovulation induction combined with IUI was associated with increased risk of preterm birth and low birth weight.

However, other studies did not describe such association (Huttunen et al., 1999; Hoy et al., 1999).

In our study live birth at term was $69.3 \%, 11.5 \%$ was preterm , $15.4 \%$ end with spontaneous abortion and one case of ectopic pregnancy we conclude that for infertile couples in which the women has no identifiable infertility factor and the man has motile sperm. The combination of ovulation induction and IUI is an effective means of achieving pregnancy.

For couples having difficulty achieving pregnancy unless both tubes are completely blocked, there is no sperm or the women never ovulate. The chance of achieving a pregnancy is not zero.

And we cannot exclude the possibility that exposure to more cycles of IUI could have led to higher live birth rate.

\section{CONCLUSION}

Intrauterine insemination is useful for the treatment of infertility in women with unexplained causes of infertility.

\section{REFERENCES}

Abdelkader, A.M. and J. Yen, 2009. The potential use of intrauterine insemination as a basic option for infertility. Rev. Technol. Int. 11: 609-622. ISBN: 1315021461

Ahinko-Hakamaa, K., H. Hubtala and H. Tinkanen, 2007. Success in intrauterine insemination: The role of etiology. Acta Obstetricia. Gynecol., 86: 855-860. DOI: $10.1080 / 00016340701416895$

Andersen, A.N., V. Goossens and A.P. Ferraritti, 2008. Assisted reproductive technology in Europe hum. Report, 23: 756-771. DOI: 10.1093/humrep/den014

Bhattacharya, S., J. Mollison, S. Worth, C. Tay and A. Harrold, 2008. Clomifene citrate or unstimulated intrauterine insemination compared with expectant management for unexplained infertility: Pragmatic randomized controlled trial. B.M. J., 337: 716-728. DOI: 10.1136/adc.2007.126367
Bilijan, M.M., M. Nen and T. Tulandi, 1999. Prospective randomized double trial of the correlation between time administration and antiestrogenic effects of clomifene citrate on reproductive end organs. Fertile. Steril., 71: 633638. ISBN: 0572014825

Boomsma, C.M., M.J. Heineman and B.J. Cohlen, 2007. Farquharc. Semen preparation techniques for intrauterine insemination. Cochrane Database Syst. Rev. 7: 1223-1235. DOI: 10.1136/adc.2007.125641

Brosens, I., S. Gordts, M. Valkenbury and P. Puttemans, 2004. Investigation of the infertility couple: When is the appropriate time to explore female infertility. Hum. Reproduct., 19: 16891692. DOI: $10.1093 /$ humrep/deh314

Collins, J., 2003. Current best evidence for the advanced treatment of unexplained subfertility. Hum. Report, 18: 907-912. DOI: 10.1093/humrep/deg170

Crosignani, P.G. and F.O. Maggiore, 2009. Intrauterine insemination. Hum. Reproduct. Update, 15: 265277. DOI: $10.1093 /$ humrep/den381

Gandoin, M., R. Dobbie and A. Finlayson, 2003. Ovulation induction intrauterine insemination in infertility couples is associated with low birth weight infants. Am. J. Obstet Gynecol., 188: 611616. DOI: $10.1067 /$ S0002-9378(03)00299-0

Hoy, J., A. Venn, J. Halliday and G. Kovacs, 1999. Perenatal and obstetric outcomes of donor insemination using cryopreserved semen in Victoria. Australia. Hum. Reproduct., 14: 17601764. DOI: $10.1093 /$ humrep/14.7.1760

Huttunen, S.N., M. Gissler and H. Mortikanen, 1999. Obstetric and perinatal outcome of pregnancies after intrauterine insemination. Hum. Report, 14: 2110-2115. DOI: 10.1093/humrep/14.8.2106

Keddy, U.M., R.G. Wapner, R.W. Rebar and R.I. Jasca, 2007. Infertility, assisted reproductive technology and adverse pregnancy outcomes: Executive summary of a national institute of child health and Human development workshop. Obstetrics Gynecol., 109: 967-977. DOI: 10.1097/01.AOG.0000259316.04136.30

Klein, J., D. Kuleta and J.C. Petrozza, 2007. Intrauterine Insemination (IUI) outcomes in cases performed by nurses as compared with cases performed by physicians. Fertility Sterility, 88: 8991. DOI: 10.1016/j.fertnstert.2006.05.073

Oddene, B.J., I. Jonkelaa and H.N. Huyse, 1999. Psychosocial experiences in women facing fertility problems. Hum. Reproduct., 14: 255-261. ISBN: 0754315401 
Omloelet, W., K. Deblaere and E. Bosmans, 2003. Semen quality and intrauterine insemination. Reproduct. Biomed. Online, 7: 485-492. DOI: 10.1016/j.fertnstert.2011.01.151

Overstreet, J.W., 1997. Brazil C. Semen Analysis. In: Infertility in Male, Lipshultz L.I. and S.S. Howards (Eds.). St Louis: Mosby-Year Book, pp: 487-490. New York. ISBN: 978-0-7817-6886-3

Royal College of Obstetricians and Gynaecologists, 1998. The management of infertility in secondary care; evidence based guid lines No.3. London.
Stephen, E.H. and A. Chandra, 2000. Hse of infertility services in the united states. Family Plann. Perspective, 132: 132-137. ISBN: 978-0-32081542-1

Temploton, A., 1995. Infertility, epidemiology, a etiology and effective management. Health Bull. Edimb., 53: 294-298. ISBN: 0-521-03176-6

Voorhis, V., A.F. Sparks and B.D. Allen, 1997. Costeffectiveness of infertility treatment. Fertile. Steril., 67: 830-860. ISBN: 1749-6632 